九州大学学術情報リポジトリ

Kyushu University Institutional Repository

\title{
NEW SYNONYMIES AND NEW RECORDS OF SOME COSMOPOLITAN SPECIES OF THE GENUS TETRAGNATHA (ARANEAE : TETRAGNATHIDAE)
}

Okuma, Chiyoko

https://doi.org/10.5109/2437

出版情報 : ESAKIA. 20，pp.69-80，1983-12-15. Entomological Laboratory，Faculty of Agriculture， Kyushu University

バージョン:

権利関係 : 


\title{
NEW SYNONYMIES AND NEW RECORDS OF SOME COSMOPOLITAN SPECIES OF THE GENUS TETRAGNATHA \\ (ARANEAE : TETRAGNATHIDAE)*
}

\author{
C ніуоко О кUM A \\ Entomological Laboratory, Faculty of Agriculture, \\ Kyushu University, Fukuoka 812, Japan
}

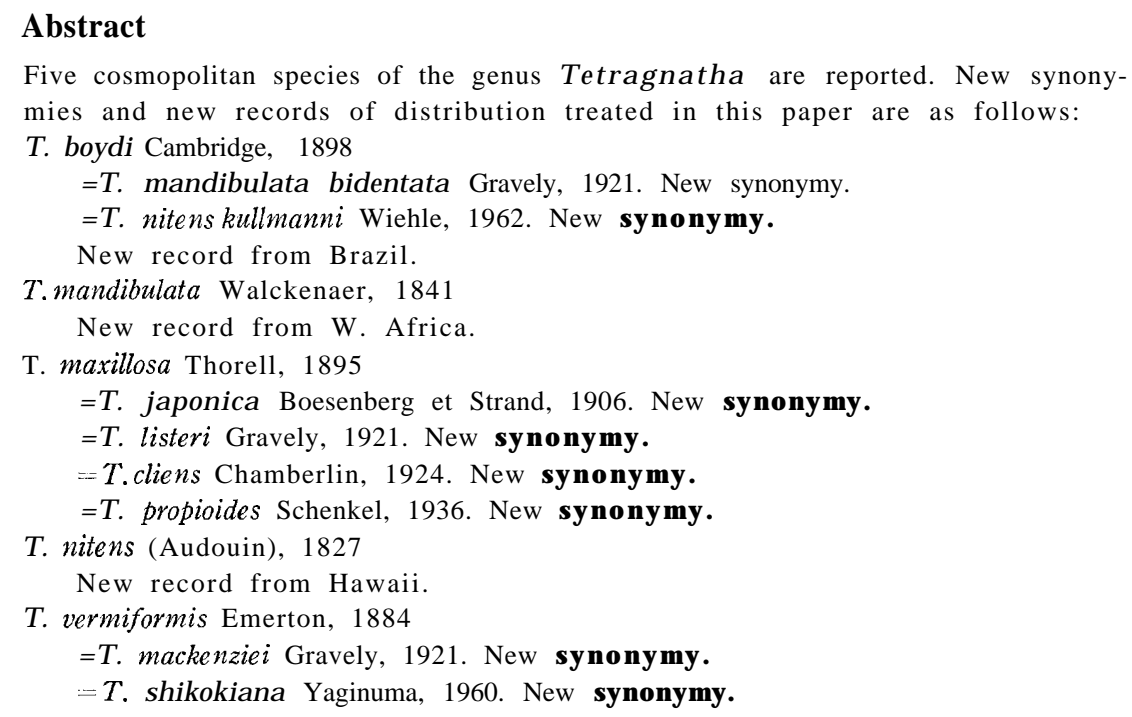

This paper reports five species of the genus Tetragnatha which were collected from various parts of the tropics to temperate regions by many seniors and other colleagues. In the present paper, I redescribe and illustrate the five species and I would like to remark here that the measurements of relative length of legs are expressed as a good character for separating species. New synonymies are proposed and new collecting records are also presented. The specimens examined in the present study are preserved in the collection of the Entomological Laboratory, Faculty of Agriculture, Kyushu University.

* Contribution from the Entomological Laboratory, Faculty of Agriculture, Kyushu University, Fukuoka (Ser. 3, No. 133). 
Before going further, I wish to express my hearty thanks to Prof. Y. Hirashima of Kyushu University for the helpful suggestions and encouragement throughout the course of this work. I heartily thank many colleagues who offered me specimens for the present study, especially to Dr. H. D. Catling, Dr. Paul Cochereau, Dr. C. Cokendolpher, Prof. Y. Hirashima, Dr. N. Hokyo, Dr. J. Martens, Dr. H. Mita, Dr. K. Morimoto, Dr. H. Murakami, Dr. T. Nishida, Dr. Y. Nishikawa, Dr. A. Otake, Dr. 0. Tadauchi, and Dr. K. Umeya.

\section{Tetragnatha boydi Cambridge}

Tetragnatha boydi Cambridge, 1898, Proc. Zool. Soc. London., 389 (Socotra) ; Lessert, 1915, Rev. Suisse Zool., 23(1): 14 (E. Africa); Lawrence, 1936, Ann. Transv. Mus., 17(2): 152 (S. Africa) ; Roewer, 1942, Katalog der Araneae, 2: 978.

Tetragnatha praedator Tullgren, 1910, Aran. in: Sjöstedt Kilimand. Exped., 3(20:6): 147 (E. Africa).

Tetragnatha nitens Lawrence, 1927, Ann. S. Afr. Mus., 25(1): 27 (S. W. Africa).

Tetragnatha mandibulata bidentata Gravely, 1921, Rec. Ind. Mus., 22: 422 (India) ; Tikader, 1966, J. Assam Sci. Soc., 9: 3 \& 9 (India). New synonymy.

Tetragnatha nitens kullmanni Wiehle, 1962, Senck. biol., 43(5): 377 (Sardinia). New synonymy.

Specimens examined: $4 \precsim \precsim$ and 5 우, W. Africa, Bouaké. VII. 1977, Los Sun Ly leg.; 1 오. Nepal, Kathmandu, Vally Chauni, 1300-1400 m, 17. IX. 1969, J. Martens leg.;2우우, Nepal, Jiri, 1800-1900 m, 10. IV. 1973, J. Martens leg. ; 5 우우, Nepal, Thanaphedi, 1500-1550 m, foot of Mt. Siwapuri, 8. X. 1981, Y. Nishikawa leg. ; $1 \delta^{`}$ and 3 우우, Nepal, Majbhatte, 1060 m, N. W. of Pokhara, 12. X. 1981, Y. Nishikawa leg.; $1 \sigma^{\star}$ and 5 우우, Brazil, Brazilia, Planaltina, 25-29. I. 1982, Y. Murakami leg.

Distribution: Africa, Brazil (new record) and Nepal.

This species was described from Socotra, an island near the east side of Somali Peninsula, and has been recorded only from Africa. All specimens from Nepal, W. Africa and Brazil very well agree with Cambridge's description and figures. The figures and the descriptions indicate that both T.mandibulata bidentata and T. nitens kullmanni are undoubtedly identical with T. boydi.

Note: This species resembles T. mandibulata in the appearance (color, size and relative length of legs) and is quite similar to $T$. nitens in the shapes of male and female chelicerae, male palp and female epiginal fold.

\section{Tetragnatha mandibulata Walckenaer}

Tetragnatha mandibulata Walckenaer, 1841, Hist. Nat. Ins. Apt., 2: 211 (Guam) ; Roewer, 1942, Katalog der Araneae, 2: 984, Chrysanthus, 1963, Nova Guinea, Zool., 24: 733 (New Guinea) ; Okuma, 1968, Mushi, 42: 101 (Thailand) ; Okuma, 1968, Actaarach., 21: 40 (Amami-Oshima, Japan); Chu \& Okuma, 1970, Mushi, 44: 73 (Taiwan) ; Chrysanthus, 1975, Zool. Verhandl., 140: 6 (New Guinea) ; Saaristo, 1978, Ann. Zool. Fenn., 15: 99 (Seychele) ; Okuma and Kishimoto, 1981, Jap. J. appl. Ent. Zool., 25: 297 (Java, Su- 


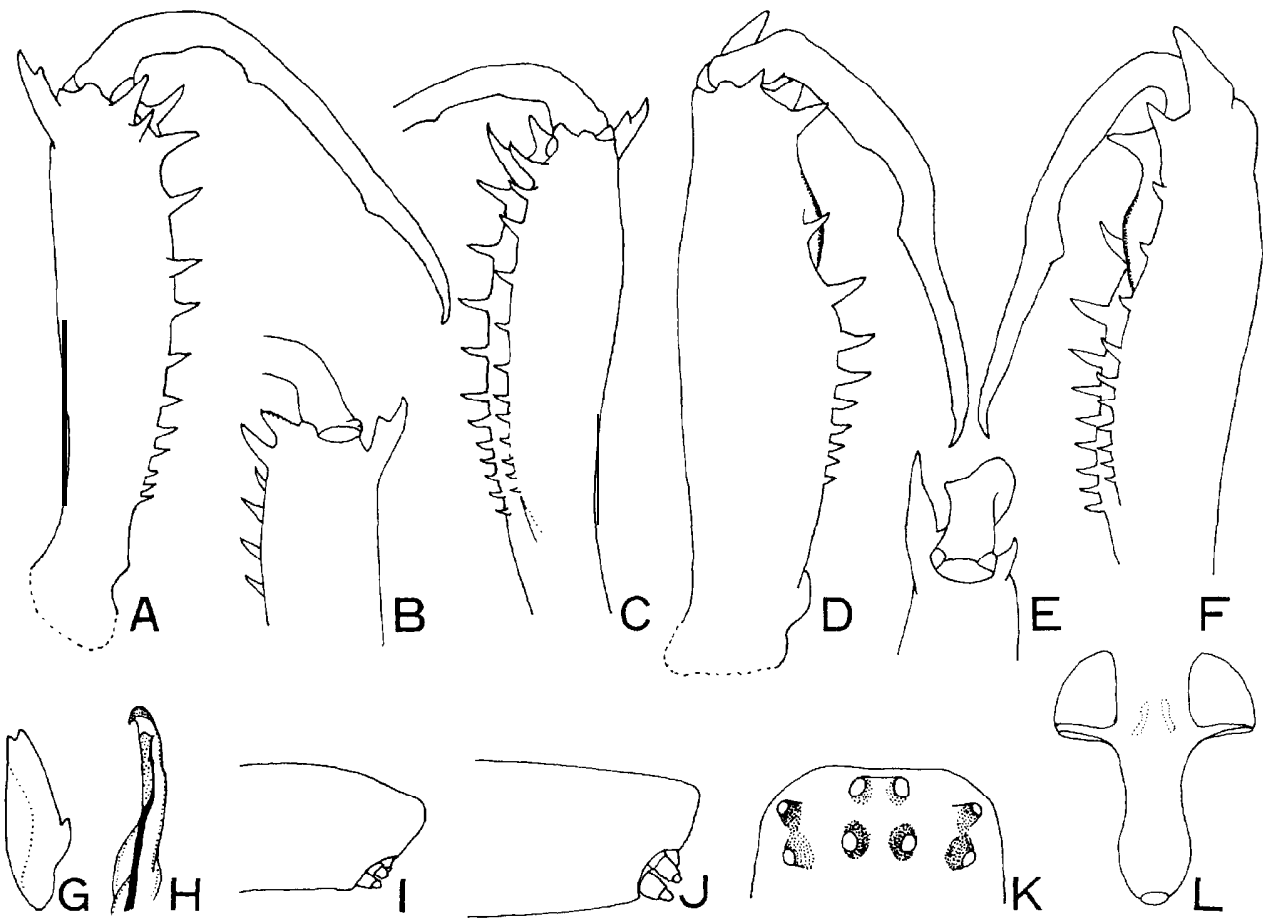

Fig. 1. Tetragnatha boydi Cambridge. A: Left chelicera of male. B: Ditto, lateral. C: Ditto, ventral. D: Left chelicera of female. E: Ditto, lateral. F: Ditto, ventral. G: Paracymbium of male. H: Distal end of conductor and embolus of male. I: Distal end of Abdomen, male. J: Ditto, female, K: Eye group of female. L: Genital fold of female.

matra, Malaysia, Thailand, Philippines, Taiwan, China and Japan).

Specimens examined : $3 \precsim \delta$ and 4 우, W. Africa, Bouaké, VII. 1977, Los Sun Ly leg.; 1 万 and 4 우, Nepal, Majbhatti, 1060 m, N. W. of Pokhara, 12. X. 1981, Y. Nishikawa leg. ;2우우, Nepal, Kiumrong, 1800 m, S. of Mt. Annapurna, 22. X. 1981, Y. Nishikawa leg. ; $11 \precsim \precsim$ and 7 우우, West Malaysia, I-II. 1976, A. Otake leg.; 1 우, Sumatra, 2. III. 1976, A. Otake leg. ; 1 ๙ , E. Java, 19. II. 1976, A. Otake leg. ; 233, Philippines, Bay Laguna, 10. VIII. 1979, K. Morimoto leg. Many other specimens of both sexes from Thailand, Taiwan and Japan (the Ryukyus) were examined.

Distribution: W. Africa (new record), S. E. Asia, Australia and Polynesia.

This species has been known to widely occur in S. E. Asia, Australia and Polynesia. This species is recorded from W. Africa for the first time in this paper. It is suggested that T.confraterna Banks, 1909 from Costa Rica may be a synonym of this species.

моте: This species is a close relative of $T$. boydi. Because this species has been often collected together with $T$. boydi at the same time and the 

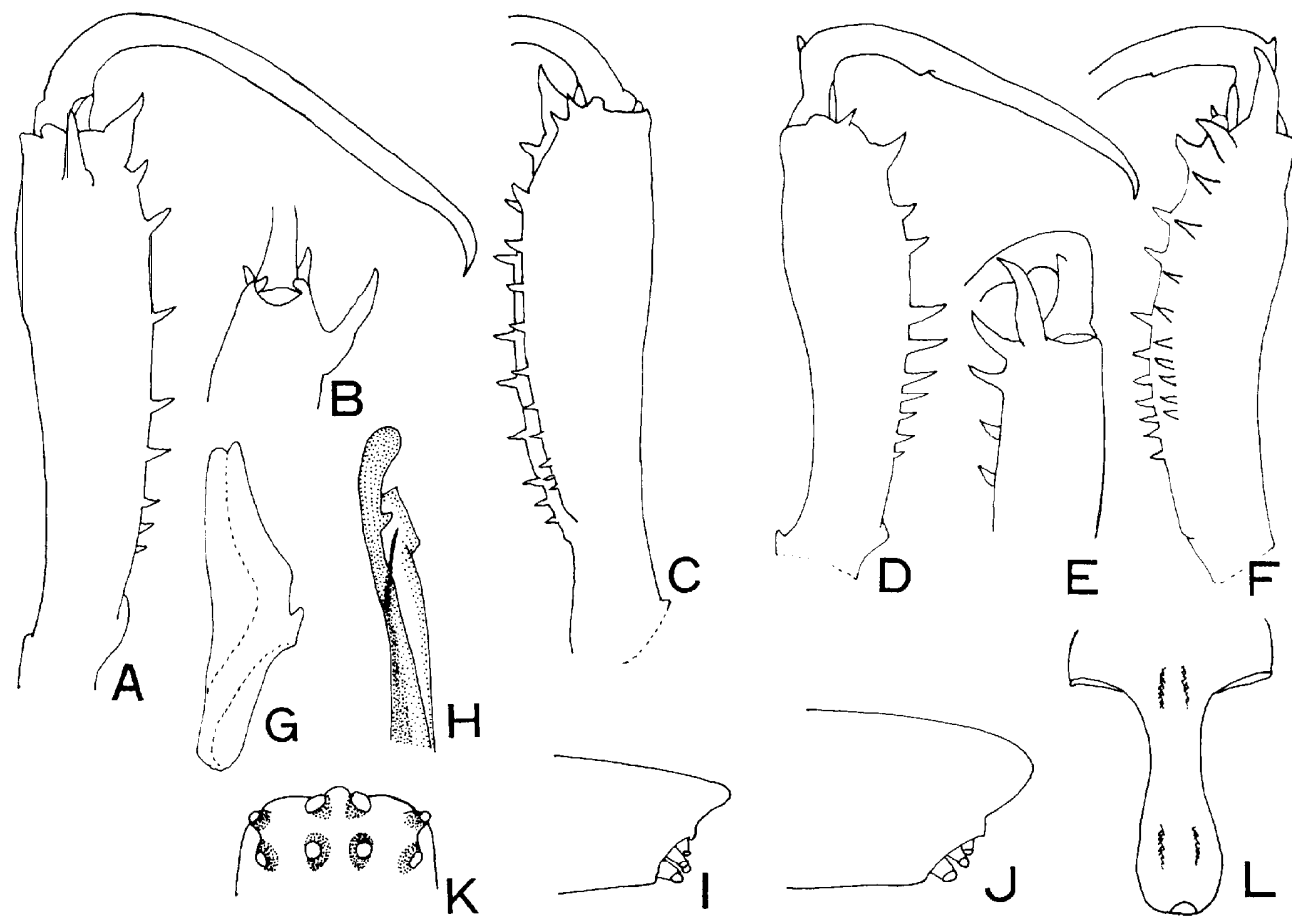

Fig. 2. Tetragnatha mandibulata Walckenaer. A: Left chelicera of male. B : Ditto, lateral. C: Ditto, ventral. D: Left chelicera of female. E: Ditto, lateral. F: Ditto, ventral. G: Paracymbium of male. H: Distal end of conductor and embolus of male. I: Distal end of abdomen, male. J: Ditto, female. K: Eye group of male. L: Genital fold of female,

same place in W. Africa and $\mathrm{Nepal}$, specific identification requires careful examination.

\section{Tetragnatha maxillosa Thorell}

Tetragnatha maxillosa Thorell, 1895, Descr.Catal. Spid. Burma.: 139 (Burma and W. Malaysia) ; Roewer, 1942, Katalog der Araneae, 2: 974; Chrysanthus, 1975, Zool. Verhandl., 140: 8 (New Guinea).

Tetragnatha mandibulata Thorell, 1890, Ann. Mus. Civ. Genova, 28: 221 (Java).

Tetragnatha japonica Boesenberg et Strand, 1906, Abh. Senckenbg. Ges., 30(1-2): 177 (Japan); Saito, 1933, Trans. Sapporo Nat. Hist. Soc.,13(1): 46 (Taiwan) ; Uemura, 1936, Acta arach., 1(3) : 82 (Japan) ; Yaginuma, 1941, Acta arach., 6(4): 127 (Taiwan) ; Paik, 1953, Atypus, 4: 20 (Korea) ; Yaginuma, 1960, Spiders of Japan in Colour,: 73 (Japan) ;Okuma, 1968, Mushi, 42: 99 (Thailand) ; Song et al., 1976, Zool. Mag., 4: 38 (China) ;Okuma and Kishimoto, 1981, Jap. J. appl. Ent. Zool., 25: 297 (Sri Lanka, Java, Sumatra, Malaysia, Thailand, Philippines, Taiwan, China. Japan, Korea and Himalaya). New synonymy.

Tetragnatha listeri Gravely, 1921, Rec. Ind. Mus., 22: 443 (Sri Lanka, Nepal, India, Burma, W. Malaysia and Thailand). New Synonymy.

Tetragnatha cliens Chamberlin, 1924, Proc. U. S. Nat. Mus., 63(13): 12 (S. China). New 
synonymy.

Tetragnatha propioides Schenkel, 1936, Ark. Zool., 29: 89 (Szechwan, China). New synonymy.

Table 1. Tetragnatha boydi Cambridge. Relative length of legs.

\begin{tabular}{|c|c|c|c|c|c|c|c|c|}
\hline Locality & & $\mathrm{n}$ & $\begin{array}{l}\text { Length } \\
\text { mean } \pm \text { s. d. }\end{array}$ & $\begin{array}{l}\text { of } 1 \text { st leg } \\
\text { range }\end{array}$ & $\begin{array}{c}\text { 1st leg } \\
\text { ratio }\end{array}$ & $\begin{array}{l}\text { 2nd leg } \\
\text { ratio(r) }\end{array}$ & $\begin{array}{l}\text { 3rd leg } \\
\text { ratio(r) }\end{array}$ & $\begin{array}{l}\text { 4th leg } \\
\text { ratio(r) }\end{array}$ \\
\hline Africa & 3 & 4 & $29.45 \pm \mathbf{1 . 4 8}$ & $27.23-30.25$ & 100 & $62 \quad(0.993)$ & $27(0.975)$ & $56 \quad(0.985)$ \\
\hline Brazil & & 1 & 33.80 & & & 60 & 26 & 56 \\
\hline Nepal & & 1 & 26.80 & & " & 62 & 27 & 57 \\
\hline Africa & क & 5 & $27.34 \pm 3.73$ & $23.80-32.70$ & " & $-63(1.000)$ & $28(0.984)$ & $56(0.996)$ \\
\hline Brazil & & 5 & $28.48 \pm 2.70$ & $25.93--33.05$ & "I & $62(0.991)$ & $28 \quad(0.985)$ & $57(0.981)$ \\
\hline Nepal & & $11 \mid$ & $31.91 \pm 3.73$ & $24.78-34.80$ & " & $63(0.998)$ & $28 \quad(0.992)$ & $60 \quad(0.999)$ \\
\hline
\end{tabular}

Table 2. Tetragnathamandibulata Walckenaer. Relative length of legs.

\begin{tabular}{|c|c|c|c|c|c|c|c|}
\hline Locality & n & $\begin{array}{l}\text { Length } \\
\text { mean } \pm \text { s. d. }\end{array}$ & $\begin{array}{l}\text { of } 1 \text { st leg } \\
\text { range }\end{array}$ & $\begin{array}{l}\text { st leg } \\
\text { ratio }\end{array}$ & $\begin{array}{l}\text { 2nd leg } \\
\text { ratio(r) }\end{array}$ & $\begin{array}{l}\text { 3rd leg } \\
\text { ratio(r) }\end{array}$ & $\begin{array}{l}\text { 4th leg } \\
\text { ratio(r) }\end{array}$ \\
\hline Japa n & 3 & $30.28 \pm 2.98$ & $26.45-33.45$ & 100 & $61(0.991)$ & $27(0.970)$ & $59(0.994)$ \\
\hline Taiwan & 10 & $28.89 \pm 3.09$ & $26.05-33.35$ & & 59 (0.990)' & $25(0.997)$ & $58(0.986)$ \\
\hline Thailand & d 110 & 29.4814 .15 & $22.10-33.80$ & & $58(0.997)$ & $25(0.986)$ & $57(0.997)$ \\
\hline Africa & 3 & $30.18 \pm 1.10$ & $29.45-31.45$ & & $60(0.910)$ & $26(0.812)$ & $59(0.942)$ \\
\hline Japan & 우 9 & $32.02 \pm 3.36$ & $24.16-35.80$ & & $60(0.992)$ & $28(0.983)$ & $60(0.977)$ \\
\hline Taiwan & 10 & $24.57 \pm 2.19$ & $21.60-28.60$ & $" \prime$ & $60(0.995)$ & $27(0.830)$ & $59(0.991)$ \\
\hline Thailand & 10 & $31.77 \pm 2.54$ & $27.45-35.80$ & & $59 \quad(\mathbf{0 . 9 6 0})^{\prime}$ & $26(0.958)$ & $58(0.966)$ \\
\hline Africa & 3 & $27.33 \pm 2.28$ & $25.25-29.95$ & & 60 (1.000)' & $27(0.987)$ & $60(0.993)$ \\
\hline
\end{tabular}

Table 3. Tetragnatha maxillosa Thorell. Relative length of legs.

\begin{tabular}{|c|c|c|c|c|c|c|c|}
\hline Locality & $\mathrm{n}$ & $\begin{array}{l}\text { Length } \\
\text { mean ts. d. }\end{array}$ & $\begin{array}{l}\text { f } 1 \text { st leg } \\
\text { range }\end{array}$ & $\begin{array}{l}1 \text { st leg } \\
\text { ratio }\end{array}$ & $\begin{array}{l}\text { 2nd leg } \\
\text { ratio }(r)\end{array}$ & $\begin{array}{l}\text { 3rd leg } \\
\text { ratio(r) }\end{array}$ & $\begin{array}{l}\text { 4th leg } \\
\text { ratio(r) }\end{array}$ \\
\hline Japan & 12 & $25.88 \pm 3.17$ & $21.85-33.40$ & 100 & $59(0.996)$ & $27 \quad(0.982)$ & $59(0.997)$ \\
\hline Taiwan & 10 & $21.75 \pm 3.67$ & $14.80-27.55$ & " & $58 \quad(0.989)$ & $26 \quad(0.974)$ & $57(0.988)$ \\
\hline Philippines & 12 & $22.62+2.31$ & $17.40-24.80$ & " & $56 \quad(0.992)$ & $25(0.977)$ & $57(0.990)$ \\
\hline Thailand & 10 & & $18.55-27.25$ & "I & $56 \quad(0.994)$ & $25 \quad(0.989)$ & $56(0.983)$ \\
\hline New Guinea & 12 & $31.16 \pm 3.31$ & $26.70-35.85$ & " & 58 (0.989) & $26 \quad(0.972)$ & $60(0.986)$ \\
\hline Japan & 12 & 26.9013 .84 & $20.30-31.75$ & " & $61(0.996)$ & $28 \quad(0.994)$ & $58(0.995)$ \\
\hline Taiwan & 10 & $24.03 \pm 2.32$ & $20.40-28.20$ & " & $61 \quad(0.995)^{\prime}$ & $27 \quad(0.989)$ & $58(0.987)$ \\
\hline Philippines & 13 & $22.54 \pm 1.43$ & $20.65-24.55$ & $" \prime$ & $59 \quad(0.980)$ & $27(0.942)$ & $56(0.971)$ \\
\hline Thailand & 10 & $24.53 \pm 2.73$ & $21.05-29.50$ & " & $58(0.994)$ & $26 \quad(0.991)$ & $55(0.998)$ \\
\hline New Guinea & a 11 & $31.49 \pm 2.98$ & $26.80--35.90$ & " & 60 (0.980); & $28 \quad(0.946)$ & $59(0.974)$ \\
\hline
\end{tabular}


Table 4. Tetragnatha nitens (Audouin). Relative length of legs.

\begin{tabular}{|c|c|c|c|c|c|c|c|}
\hline Locality & ${ }_{1}^{1} \mathrm{n}$ & $\begin{array}{l}\text { Length } \\
\text { mean } \pm \text { s. d. }\end{array}$ & $\begin{array}{l}\text { f } 1 \text { st } \text { leg } \\
\text { range }\end{array}$ & $\begin{array}{l}\text { 1st leg } \\
\text { ratio }\end{array}$ & $\begin{array}{l}\text { 2nd leg } \\
\text { ratio(r) }\end{array}$ & $\begin{array}{l}\text { 3rd leg } \\
\text { ratio(v) }\end{array}$ & $\begin{array}{l}\text { 4th leg } \\
\text { ratio(v) }\end{array}$ \\
\hline Japan & d 7 & $25.25 \pm 3.44$ & $20.30-26.40$ & 100 & $69(0.985)^{\prime}$ & $31(0.973)$ & $64(0.982)$ \\
\hline Taiwan & 10 & $23.61 \pm 4.12$ & $17.55-30.90$ & " & $68 \quad(0.998)$ & $30(0.989)$ & $63 \quad(0.997)$ \\
\hline Thailand & 10 & $25.37 \pm 4.20$ & $17.65-32.05$ & " & $67 \quad(0.998)$ & $30(0.992)^{\prime}$ & $62 \quad(0.998)$ \\
\hline Florida & 6 & $25.94 \pm 4.57$ & $22.50-30.20$ & " & $70 \quad(0.999)$ & $32(0.995)$ & $\begin{array}{ll}65 & (0.995) \\
-\end{array}$ \\
\hline Japan & \& 10 & 23.9914 .48 & $15.65-30.85$ & " & 68 (0.997); & $31 \quad(0.991)$ & $64 \quad(0.993)$ \\
\hline Taiwan & 10 & $20.64 \pm 2.88$ & $16.60-24.90$ & $\prime \prime$ & $68(0.992)$ & $31(0.982)$ & $65 \quad(0.993)$ \\
\hline
\end{tabular}

Table 5. Tetragnatha vermiformis Emerton. Relative length of legs.

\begin{tabular}{|c|c|c|c|c|c|c|c|c|}
\hline & Locality & $n$ & $\begin{array}{l}\text { Length } \\
\text { mean } \pm \text { s.d. }\end{array}$ & $\begin{array}{r}\text { of } 1 \text { st leg } \\
\text { range }\end{array}$ & $\begin{array}{l}1 \text { st } \\
\text { leg } \\
\text { ratio }\end{array}$ & $\begin{array}{l}\text { 2nd leg } \\
\text { ratio }(r)\end{array}$ & $\begin{array}{l}3 \text { rd leg } \\
\text { ratio(y) }\end{array}$ & $\begin{array}{l}\text { 4th leg } \\
\text { ratio(r) }\end{array}$ \\
\hline Japan & (Ibaragi) & 30 & $20.51 \pm 2.89$ & 14.23325 .25 & 100 & $70(0.996)$ & $31 \quad(0.967)$ & $65 \quad(0.992)$ \\
\hline Japan & (Tokushima) & 8 & 19.91-11.17 & $18.15-21.75$ & $5 \prime \prime$ & $68(0.994)$ & $29(0.869)$ & $63(0.963)$ \\
\hline China & & $8^{i}$ & $22.59 \pm 2.50$ & $18.48-25.45$ & " & $70 \quad(0.992)$ & $29(0.939)$ & $65 \quad(0.996)$ \\
\hline Texas & & 1 ' & 24.40 & & $" 1$ & 71 & 32 & 64 \\
\hline Japan & (Ibaragi) 우 & 30 & $17.21 \pm 2.50$ & $12.70-20.70$ & $0 \quad 1$ & $70(0.996)^{\prime}$ & $32(0.990)$ & $67(0.995)$ \\
\hline Japan & (Tokushima) & 8 & $15.86 \pm 0.77$ & $14.65-16.75$ & $\prime \prime$ & $68(0.978)$ & $31 \quad(0.988)$ & $66(0.988)$ \\
\hline China & & 8 & 18. 481-1. 35 & $17.10-20.95$ & $5 \prime$ & $70(0.983)$ & $31 \quad(0.919)$ & $67 \quad(0.963)$ \\
\hline Texas & & 1 & 18.10 & & $" 1$ & 172 & 32 & 67 \\
\hline
\end{tabular}

$(n)=$ The number of specimens. $(r)=$ The coefficient of correlation.

Specimens examined : $1 \delta$, Nepal, Kathmandu Valley, $1350-1400 \mathrm{~m}$, IV-V.

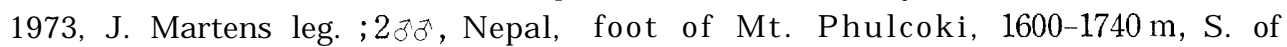
Kathmandu, 16. IX. 1981, Y. Nishikawa leg.;3 3. , Nepal, Majbhatti, 1060 m, N. W. of Pokhara, 12. X. 1981, Y. Nishikawa leg., $1 \delta^{\Uparrow}$ and 5 우우, Nepal, Kiumrong, $1800 \mathrm{~m}$, S. of Mt. Annapurna, 22. X. 1981, Y. Nishikawa leg.; $7 \Varangle ð$ and 2 오, Sri Lanka, II--III. 1973, H. Mita leg. ; 1 우, New Guinea, Wau, 22. VIII. 1969, Y. Hirashima leg.;12 8 and 11 와, New Guinea, Wau, 15-22. VIII. 1982, Y. Hirashima and 0. Tadauchi leg.; 2 우우, E. Java, 12. II. 1976, A. Otake leg. ;1우, Sumatra, 2. III. 1976, A. Otake leg.; 4우우, W. Malaysia, I-II. 1976, A. Otake leg. ; 1233 and 12 우, Philippines, Davao, 29. VIII. 1979, K. Morimoto leg. ;2 ॐ and 4 우, China, Hang Zhou, 26. VI. 1980, K. Umeya leg. Many other specimens of both sexes from Thailand, Taiwan, Korea and Japan were examined.

Distribution: Nepal, S. E. Asia, E. Asia to New Guinea.

T.maxillosa Thorell, 1895 was originally described from Java and successively recorded from Burma, Malaya and India. But this species received no attention for some time. In 1975, Chrysanthus redescribed and illustrated the species and recorded it from New Guinea for the first time. T.japonica 


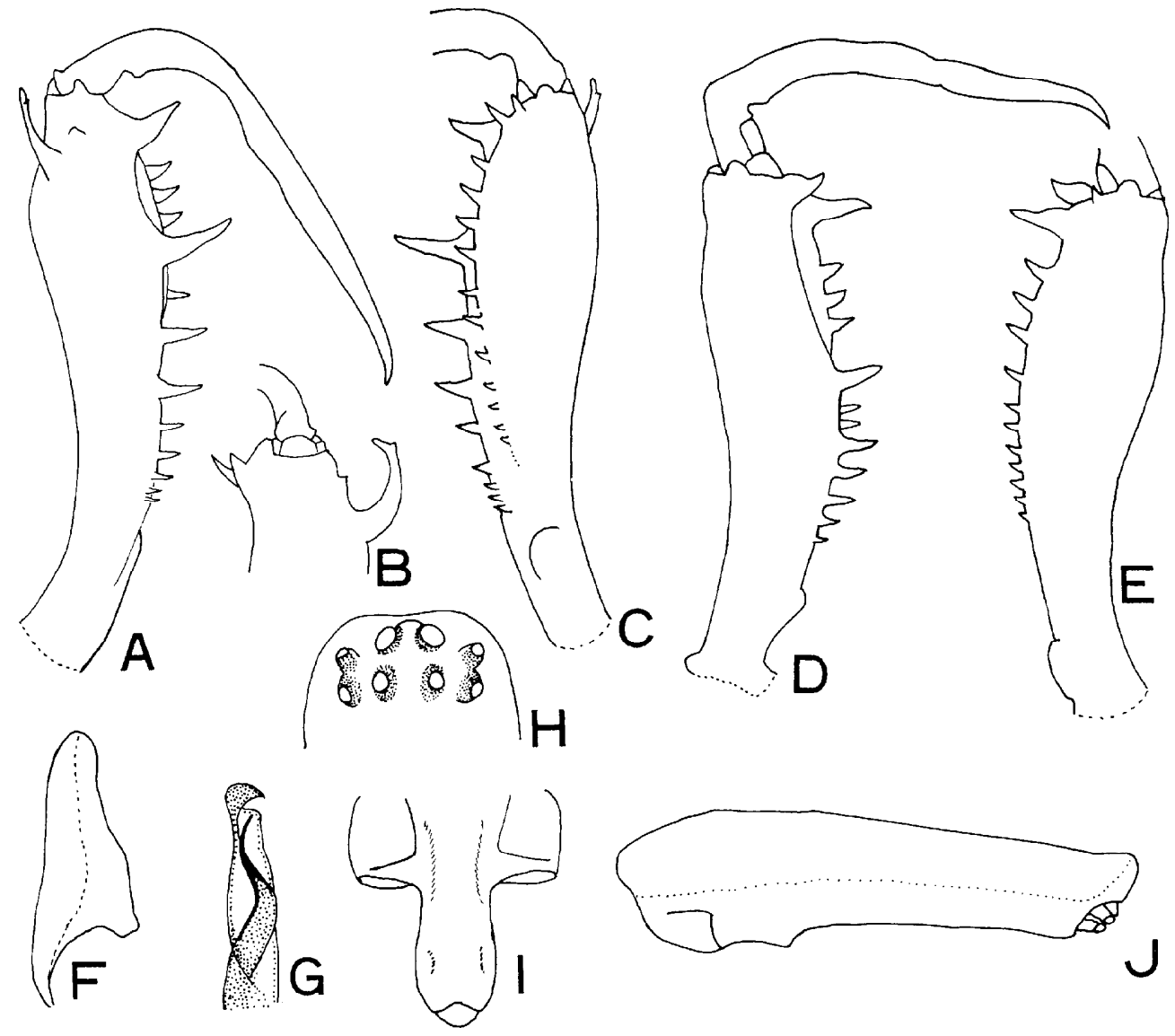

Fig. 3. Tetragnatha maxillosa Thorell. A: Left chelicera of male. B: Ditto, lateral. C: Ditto, ventral. D: Left chelicera of female. E. Ditto, ventral. F : Paracymbium of male. G: Distal end of conductor and embolus of male. H: Eye group of male.

I: Genital fold of female. J: Lateral view of abdomen, female.

Boesenberg et Strand, 1906 was described from Japan and later recorded from Korea, Taiwan, China, Thailand, Sri Lanka, Java, Sumatra, Malaysia, Philippines and Himalaya. A careful examination of many specimens mentioned above with Chrysanthus' and Thorell's descriptions of T. maxillosa has convinced me that T. japonica is undoubtedly identical with T. maxillosa. In addition, it is very certain that T. listeri, T. cliens and T. propioides are undoubtedly identical with T. maxillosa. T. strandi Lessert, 1915 from Uganda may a synonym of this species.

Tetragnatha nitens (Audouin)

Eugnatha nitens Audouin, 1827, Explic. Planch. Arachn. in: Savigny, Desc. de l'Egypt., 22: 323. 
Tetragnatha nitens Roewer, 1942, Katalog der Araneae, 1: 978; Okuma, 1968, Mushi, 42: 102 (Thailand) ; Okuma, 1968, Actaarach., 21: 40 (Japan (Ishigaki \& Okinawa) and Taiwan) ; Song et al., 1976, 2001, Mag., 4: 39 (China) ; Levi, 1981, Bull. Mus. Comp. Zool.,149(5): 291 (Neotropics).

Specimens examined: $6 \gtrsim$, Florida, New Nans' Lake, 23. VI. 1978, J. Cokendolpher leg. ;1ð̋, Texas, Wichita Co., 17. X. 1972, Zaltsberg leg. ;4우우, Hawaii, Kauai, 31. X. 1978, T. Nishida leg. Many other specimens of both sexes from Japan, Taiwan and Thailand were examined.

Distribution : This is a pansubtropical and pantropical species. This is newly recorded from Hawaii.

This species was described from Egypt and has been known to widely occur in Africa, S. E. Asia, E. Asia and Australia. Recently Dr. Levi (1981) reported that T. antillana Simon, Eugnatha pelusia Audouin in Savigny, T. andina Taczanowski, T. vicina Simon, T. seminola Gertsch, T. peninsulana Banks, $T$.

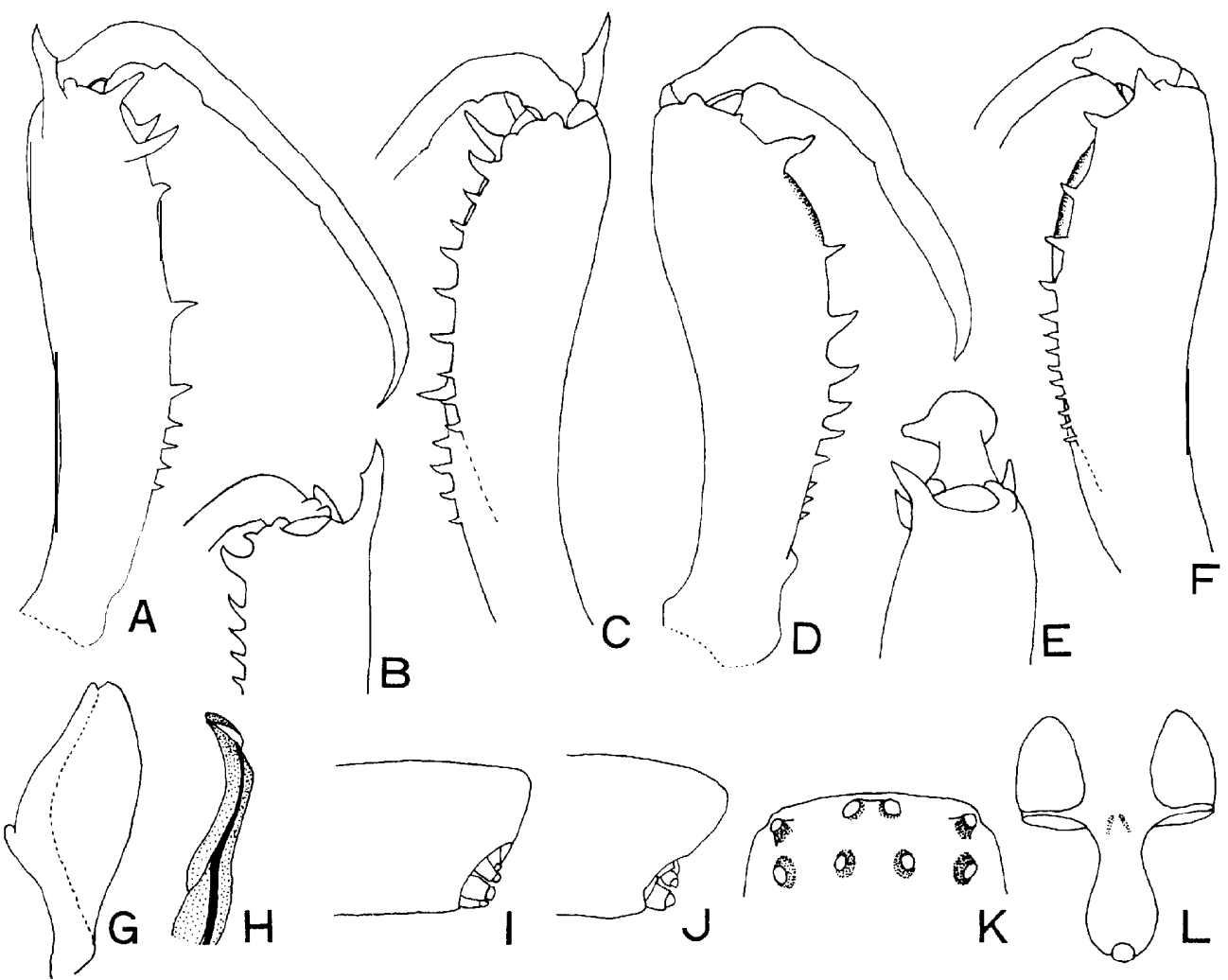

Fig. 4. Tetragnatha nitens (Audouin). A: Left chelicera of male. B: Ditto, lateral. C: Ditto, ventral. D: Left chelicera of female. E: Ditto, lateral, F: Ditto, ventral. G: Paracymbium of male. H: Distal end of conductor and embolus of male. I: Distal end of abdomen, male. J: Ditto, female. K: Eye group of female. I,: Genital fold of female. 
galapagoensis Banks, T. eremita Camberlin, T. steckleri Gertsch, T. elmora Chamberlin and T. festina Bryant (most of them from the Neotropics) are synonyms of $T$. nitens. Judging from the figures and the description of T.boeleni Chrysanthus, 1975 from New Guinea, it is considered to be identical with $T$. nitens.

Note: This species is conspicuous by the spur and the two contiguous teeth on the chelicera of the male and the diagnostic posterior cusp on the fang of the female. These characters are very similar to those of $T$. boydi, so that the identification requires careful examination, but this species is easily distinguished from $T$. boydi by the relative length of the legs (see Tables 1 and 4).

Tetragnatha vermiformis Emerton

Tetragnatha vermiformis Emerton, 1884, Trans. Connect. Acad. Sci., 6: 333 (Costa Rica) ; Seeley, 1928, Bull. New York State Mus., 278; 138 (Nebraska) ; Roewer, 1942, Katalog der Araneae, 1: 994; Kaston, 1948, Bull. Connect. State Geol. Nat. Hist. Surv., 70 : 272 (Connecticut) ; Chickering, 1957, Bull. Mus. Comp. Zool., 116: 349 (Panama) ;Chickering, 1959, Bull. Mus. Comp. Zool., 119: 495 (Michigan) ; Levi, 1981, Bull. Mus. Comp. Zool., 149: 316 (Mexico).

Tetragnatha mackenziei Gravely, 1921, Rec. Ind. Mus., 22: 438 (India and Burma) ; Shinha, 1951, Rec. Ind. Mus., 49: 79 (India) ; Okuma, 1968, Mushi, 42: 101 (Thailand) ; Okuma and Kishimoto, 1981, Jap. J. appl. Ent. Zool., 25: 297 (Sri Lanka, Thailand and India). New synonymy.

Tetragnathashikokiana Yaginuma, 1960, Spiders of Japan in Colour,: 74 (Japan) ; Song et al., 1976, Zool. Mag., 4: 38 (China) ; Okuma et al., 1978, Esakia, 11: 83 (Korea) ; Okuma and Kishimoto, 1981, Jap. J. appl. Ent. Zool., 25: 297 (China, Korea and Japan). New synonymy.

Specimens examined: $1 \delta$ and 1 , 9 , Texas, Lake Wichita, Wichita Co., 1. VII. 1977, L. K. Qonglss leg.; $1 \delta^{\star}$, Sri Lanka, II-III. 1973, H. Mita leg. ;1 Thailand, Ban-Saun Rit, 5. XI. 1966, C. Okuma leg., $2 \mho^{\star}$ and 1 young, Thailand, Lampoon, 9. XI. 1966, C. Okuma leg. ; 1 으, Thailand, Sanpatong, 3-6. XI. 1970, C. Okuma leg. ; $43 ð$ and 13 q早, Korea, Suwon, VI-IX. 1975, N. Hokyo leg.; 883 and 10 pq, China, Hang Zhou, 26. VI. 1982, K. Umeya leg.; 1838 and 15 우, Japan, Shikoku, 28-29. VIII. 1973, C. Okuma leg. ;5 $38^{\jmath}$ and 4 우우, Japan, Tokyo, 24. VIII. 1974, C. Okuma leg. ; 383 and 6 웅, Japan, Fukuoka, 15. VII. 1978, K. Ohara leg.; $30 \gtrsim$ and 30 우, Japan, Ibaragi, 22. VII. 1982, C. Okuma leg.; 3 우, Japan, Kyoto, 22. VIII. 1982, C. Okuma leg.

Distribution: India, Sri Lanka, Burma, Thailand, China, Korea, Japan and U.S. A.

T. vermiformis Emerton, 1884 was described from Barro Colorado Is. Since then, it has been known from throughout southern Canada, northwest eastern states to the Canal Zone. T. mackenziei Gravely, 1921 was described from India and has been recorded from Burma, Thailand and Sri Lanka. T. shikokiana Yaginuma, 1960 was described from Japan and has been recorded from 


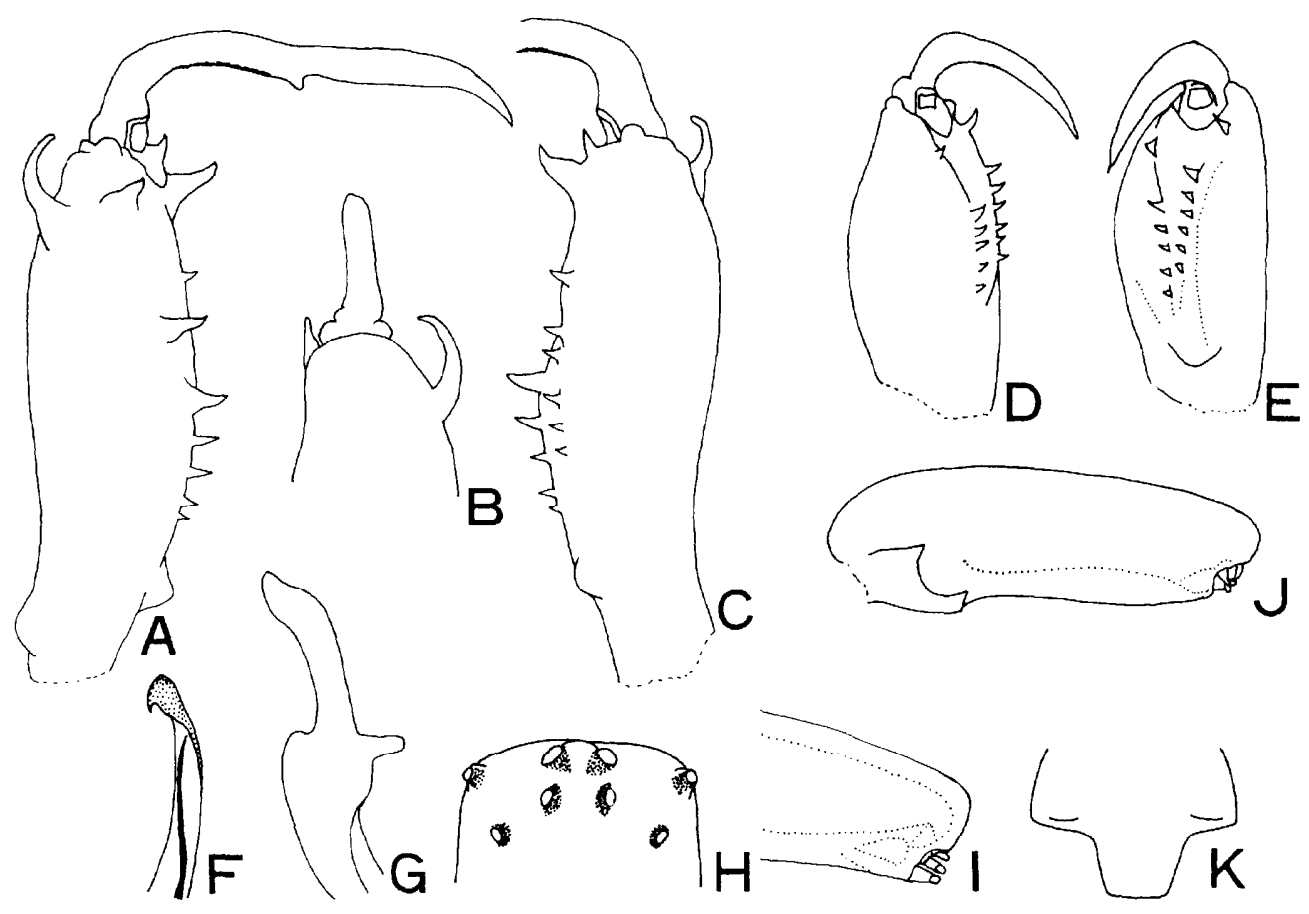

Fig. 5. Tetragnatha vermiformis Emerton. A: Left chelicera of male. B: Ditto, lateral. C: Ditto, ventral. D: Left chelicera of female. E: Ditto, ventral. F: Distal end of conductor and embolus of male. G: Paracymbium of male. H: Eye group of male. I: Distal end of abdomen, male. J: Lateral view of abdomen, female. K: Genital fold of female.

Korea and China. Careful examinations of many specimens mentioned above from Japan, China, Korea, Thailand and U. S. A. (Texas), and of several descriptions and figures of T.vermiformis, have convinced me that all of them belong to but one species. It is also suggested that T.andonea Lawrence, 1927 from S. W. Africa may be a synonym of T.vermiformis.

Nоте: This species mainly inhabits rice fields in Asia, being extremely abundant in some localities, although it is said to be uncommon in U. S. A.

\section{References}

Audouin, V. 1827. Explication Sommaire des Planchesd'Arachnides, in : J. C. Savigny, Descr. de l'Egypt., 22: 291-430.

Banks, N. 1909. Arachnida from Costa Rica. Proc. Acad. Nat. Sci. Philad., 61 : 194-234.

Boesenberg, W. \& E. Strand 1906. Japanische Spinnen. Abh.Senckenbg. Nat. Ges., 30(1-2): 93-373, T. 3-16.

Cambridge, 0. P. 1898. On a Collection of Insects and Arachnids made in Socotra. Proc. Zool.Soc. London, (1898) : 387-391, T. 31.

Chamberlin, R. V. 1924. Descriptions of New American and Chinese spiders. Proc. U. S. N ut. Mus., $63(13):$ I-38. 
Chickering, A. M. 1957. The Genus Tetragnatha (Araneae, Argiopidae) in Panama. Bull. Mus. Comp. Zool., 116: 301-354.

- 1959. The Genus Tetragnatha (Araneae, Argiopidae) in Michigan. Bull. Mus. Comp. Zool., 119 : 475-499.

Chrysanthus, Fr. 1963. Spiders from South New Guinea V. Nova Guinea, Zoology, 4: 727 750.

- 1975. Further notes on the spiders of New Guinea II (Araneae, Tetragnathidae, Theridiidae). Zool. Verhandl., Leiden, 140: 3-50.

Chu, Y.-I. \& C. Okuma 1970. Preliminary survey on the spider-fauna of the paddy fields in Taiwan. Mushi, 44(9): 65-88.

Emerton, J. H. 1884. New England spiders of the family Epeiridae. Trans. Connecticut Acad., 6: 295-341.

Gravely, F. H. 1921. Some Indian spiders of the subfamily Tetragnathinae. Rec. Ind. Mus., 22: 423-459.

Kaston, B, J. 1948. Spiders of Connecticut. Bull. Connecticut State Geol. Nat. Hist. Survey, 70: $1-874$.

Lawrence, R. F. 1927. Contributions to a knowledge of the fauna of South-West Africa. I. Ann. S. Afr. Mus., 25(1): 1-75, T. 1-4, F. 1-90.

- 1936. Spiders in: Scientific Results of the Vernay-Lang Kalahari-Expedition. Ann. Transv. Mus.,17(2): 145-158.

Lessert, R. de 1915. Arachnides de l'Ouganda et de I'Afrique Orientale Allemanda. Rev. Suisse Zool.,23(1): 1-89.

Levi, H. W. 1981. The American orb-weaver genera Dolichognatha and Tetragnatha north of Mexico (Araneae: Araneidae, Tetragnathinae). Bull. Mus. Comp. Zool., 149(5):271318.

Okuma, C. 1968. Two spiders new to fauna of Japan. Acta arach., 21(2): 40-42.

- 1968. Preliminary survey on the spider-fauna of the paddy field in Thailand. Mushi, 42(8) : 89-118.

— \& R. Kishimoto 1981. Air borne spiders collected over the East China Sea. Jap. J. appl. Ent. Zool., 25: 296-298.

- M. H. Lee and N. Hokyo 1978. Fauna of spiders in a paddy field in Suweon, Korea. Esakia, (11) : 81-88.

Paik, K. 1953. A list of spiders from Quelpart I., Korea. Atypus, (4) : 17-21.

Roewer, C. Fr. 1942. Katalog der Araneae I. 1040 pp. Bremen.

Saito, S. 1933. Notes on the spiders from Formosa. Trans. Sapporo Nat. Hist. Soc., 13(1): $32-61$.

Saaristo, M. I. 1978. Spiders (Arachnida, Araneae) from the Seychelle Islands, with notes on taxonomy. Ann. Zool. Fennici, 15: 99-126.

Schenkel, E. 1936. in Schwedisch-chinesische wissenschaftliche Expedition nach den nordwestlichen Provinzen Chinas. Ark. Zool., Stockholm, 29(A:1): 1-314.

Seely, R. M. 1928. Revision of the spider genus Tetragnatha. Bull. New York State Mus., $278: 99-149$.

Sinha, T. B. 1951. Some Indian spiders of the family Argiopidae. Rec. Ind. Mus., 49: 67-88.

Song, D. X. et al. 1976. The common species of Tetragnatha from the paddy field of Zhejiang Province. Zool. Mag., 4: 38-39. (In Chinese)

Thorell, T. 1980. Studi sui ragni Malesi e Papuani IV (1). Ann. Mus. Civ. Genova, 28: 1419.

1895. Description Catalogue of the Spiders of Burma. $406 \mathrm{pp}$. London.

Tikader, B. K. 1966. Studies on spider fauna of Khasi and Jaintia Hills, Assam, India. J. Assam Sci. Soc., 9: l-16.

Tullgren, A. 1910. Arachnoidea in :Sjöstedt, Y., Kilimandiaro-Meru Expedition (Stockholm), 
3 (20:6) : 86-172.

Uemura, T. 1936. Spiders of Wakayama Pref. in Japan. Acta arach., 1(3) : 82-100. (In Japanese)

Walckenaer, C. A. de 1841. Histoire Naturelle des Insects Aptères, 2: 548 pp. Paris.

Wiehle, H. 1962. Eine Unterart von Tetragnatha nitens ([Savigny \&] Audouin) aus Sardinien (Arach., Araneae). Senck. biol., 43(5): 377-383.

Yaginuma, T. 1941. Spiders of Formosa (1). Acta arach., 6(4): 127. (In Japanese)

1960. Spiders of Japan in Colours. 186 pp. Hoikusha, Osaka. (In Japanese) 